\title{
Distinctive brand cues and memory for product consumption experiences
}

\author{
Luk Warlop $^{\mathrm{a}, *}$, S. Ratneshwar ${ }^{\mathrm{b}}$, Stijn M.J. van Osselaer ${ }^{\mathrm{c}}$ \\ ${ }^{a}$ Katholieke Universiteit Leuven, Department of Applied Economics, Naamsestraat 69, 3000 Leuven, Belgium \\ ${ }^{\mathrm{b}}$ University of Missouri-Columbia, Bailey K. Howard World Book Chair of Marketing, Columbia, MO 65211, USA \\ ${ }^{\mathrm{c}}$ Erasmus University, P.O. Box 1738, 3000 DR Rotterdam, The Netherlands
}

\begin{abstract}
Consumer welfare depends upon how well people learn and remember quality differences among competing products. Although some researchers have argued that consumers are quite good at learning from actual consumption experience, such learning is complicated by delays between learning episodes and by delays between learning and use of the information. In the present research, we examine consumer experiential learning from a memory perspective. In a series of three taste-test studies we investigate whether and how distinctive brand names and packaging may facilitate the learning of intrinsic quality differences among products. We discuss the implications of our results for consumer decision theory, brand equity management, and trademark policy.
\end{abstract}

(C) 2004 Elsevier B.V. All rights reserved.

Keywords: Consumer learning and memory; Consumer judgments; Brand image; Brand equity; Trademarks

\section{Introduction}

Successful brands and brand equity depend upon the creation of high levels of brand awareness and distinctive as well as positive brand images (Keller, 1998; Krishnan, 1996). Prior research on brand awareness is extensive, and such work has primarily investigated consumers' ability to retrieve brands as memory targets for consideration sets or to identify

* Corresponding author. Tel.: +32 163269 41; fax: +32 1632 6732.

E-mail address: luk.warlop@econ.kuleuven.ac.be (L. Warlop). products in busy perceptual fields such as grocery store aisles (e.g., Keller, 1991). The research presented here, on the other hand, focuses on the brand image component of brand equity.

Brand image refers to the role of brand names and other aspects of a brand's trade dress as cues that retrieve or signal product attributes, benefits, affect, or overall quality (e.g., Erdem \& Swait, 1998; Henderson, Cote, Leong, \& Schmitt, 2003; Kirmani \& Rao, 2000). In this sense, the brand itself is not a memory target but a cue that might facilitate recall or inference of previously learned brand associations. This component of brand equity is especially important when consumers are trying to evaluate a product that is 
already in the consideration set, or one that is available in the consumer's choice environment. Although in most choice environments the brand cues (name, logo, packaging design, etc.) are available, visible, and easy to discriminate perceptually, consumers still have to rely on memory to associate these brand cues with the results of prior learning of product quality (Alba, Hutchinson, \& Lynch, 1991). Prior learning could have resulted in episodic memory traces of specific consumption experiences, but more likely in abstractions or summary evaluations, which are generally easier to remember than specific information (Chattopadhyay \& Alba, 1988).

But how good are consumers at learning and remembering prior consumption experiences and the resulting evaluations? The general thrust of the marketing literature has been that learning from direct experience can be effective, at least compared to indirect learning from advertising or secondary sources (Smith \& Swinyard, 1982; cf. Wright \& Lynch, 1995). However, the focus of this stream of research has typically not been on learning to discriminate among different brand experiences. For effective experiential learning, consumers need to recall exactly which prior experience went with each brand. Indeed, past research has shown that discrimination learning might be difficult even if the differences between competing brands are real and substantial (see Hoch \& Deighton, 1989 for a review). Consumers typically buy and consume products in a category sequentially, not simultaneously. Hence, the learning process often does not permit effective comparison and contrast of alternatives (Hoch \& Ha, 1986; Pechmann \& Ratneshwar, 1992). In addition, memory for brand quality may be impeded by significant delays between consumption experiences and subsequent purchase occasions when retrieval is attempted (Alba et al., 1991; Pham \& Johar, 1997). The result may be considerable confusion in memory between various brand experiences. Confusion of very satisfactory consumption experiences with lesser experiences may lead to reduced repeat purchase and dilution of brand equity for marketers of high quality products or, alternatively, to an increase in brand equity for marketers of lower quality products.

Given the aforementioned constraints, what is the role of distinctive brand cues such as brand names and brand packaging in facilitating or impeding con- sumer learning and memory for product consumption experiences? Some researchers have argued that "meaningless" or cosmetic differentiation among brands (e.g., in packaging) hurts rather than helps consumer welfare by obscuring real product differences or the absence thereof (e.g., Resnik \& Stern, 1977). Granted, such research is not specific to the context of consumer learning. Still, it seems to ignore the possibility that such extrinsic brand cues might actually enhance the memorability of prior experiences with specific brands, thereby facilitating accurate quality discrimination and improving consumer welfare.

We report below three studies involving quality judgments based on experiential learning in taste test tasks. The first two studies investigate the role of differentiation in brand names and packaging attributes, respectively, in consumer learning of product taste quality. The third study examines whether such differentiation can facilitate learning even when product prices provide potentially misleading cues regarding true quality. Theoretical, managerial, and policy implications are discussed in the final section of the paper.

\section{Conceptual background and hypotheses}

There are at least two ways in which extrinsic brand cues can affect the retrieval of prior consumption experiences. First, it is likely that a brand element such as a brand name is not represented in memory by a single conceptual node but by multiple nodes, i.e., brand elements have distributed representations (e.g., McClelland, 2000). Similar brand cues should have more of these multiple nodes in common. The presence of any one of two similar brand cues in the choice environment will activate the common nodes, and the memory representations of consumption experiences related to both brands thereby will receive activation. Thus, the presence of more common nodes will lead to more activation of consumption experiences that really belong to other brands. As a result, there may be confusion about experiential memory targets even when there is no confusion or misidentification of the brands in question.

Second, consumers' memory representations of a brand typically include many associations such as 
product categories, consumption benefits, or semantic associations suggested by the brand name (Sen, 1999). Similar brand names may share semantic associations in memory that in turn are associated with the experiential targets of prior consumption episodes. For example, during a particular consumption experience, one brand (e.g., Almarcha orange juice) may activate a particular meaning (e.g., Spain) and that meaning becomes associated with that brand's consumption experience (e.g., a very good taste). If another brand (e.g., Cordoba orange juice) activates overlapping semantic associations (e.g., to Spain), those will also become associated with the second brand's experience (e.g., a mediocre taste). Later, the presence of either brand in a choice environment (Almarcha or Cordoba) will cause the spread of activation from the brand to the meaning (Spain), and from the meaning to two different consumption experiences (very good vs. mediocre taste). Hence, even though a consumer may not be confused about the identities of the two brands at the point of purchase (because Almarcha sounds and looks different from Cordoba), he or she might still confuse the specific consumption experiences that occurred with those two brands.

There is indeed some empirical evidence to suggest that similarity (versus distinctiveness) of brand cues might increase confusion in the recall of experiential targets. For example, although most research on recall of advertising claims does not directly manipulate similarity of cues and does not involve experiential targets, it does find worse recall of advertising claims when consumers are shown more ads for products in the same product category (Burke \& Srull, 1988; Keller, 1991) or more ads for the same brand (Burke \& Srull, 1988). In a study more directly relevant to our problem, Kumar (2000) manipulated the distinctiveness of pictorial contexts during learning and then asked subjects to recall ad claims for different advertisements by cueing them with the pictorial contexts at the point of recall. Kumar's data suggest that ad claim recall improved when pictorial contexts were less similar. However, no statistics were reported for the effect discussed here, and the results may have been partially driven by identity confusion because only one context cue was presented to each subject at recall.

Further, there are important differences between memory processes for brand claims in advertising and memory for direct consumption experiences. In comparison to verbal learning, learning from sensory experience tends to promote better memory because it is more vivid and concrete (Paivio, 1971) and involves more elaborative rehearsal (Slamecka \& Graf, 1978). Correspondingly, it is possible that direct product experiences are remembered so well that regardless of brand cue distinctiveness, there is very little scope for confusion. On the other hand, product consumption experiences tend to be easily influenced by expectations (e.g., Hoch \& Deighton, 1989; Hoch \& Ha, 1986; Pechmann \& Ratneshwar, 1992). Thus, differences in conceptual brand associations may easily color perceptions of product experiences, thereby altering their evaluation and interpretation. For example, a tropical brand name may cause a product to taste better or more exotic. Consequently, recall accuracy of actual product experiences may be poor when extrinsic cues (e.g., brand names, prices) bias sensory experiences or when experiential stimuli are experienced in the same way because similar brand names (or their intermediary associations) lead to similar expectations.

In sum, both memory theory and the findings of some prior consumer research in verbal learning suggest that distinctive (vs. similar) brand cues should facilitate recall accuracy of targets associated with these respective cues. Nonetheless, as discussed above, it is not apparent whether such findings would apply to contexts where consumers learn from direct product experience. It is an empirical question whether distinctive cues in the form of differentiated brand names will increase the accuracy of memorybased quality judgments, especially in situations where such judgments are based on prior sensory experiences. More formally:

H1. Differentiated (vs. similar) brand names should yield higher accuracy in memory-based quality judgments.

In addition to brand names per se, distinctive brand cues include those aspects of product packaging that are deemed to be unique to the brand (e.g., particular packaging shapes and/or colors; Simonson, 1993). Keller (1998) suggests that developing a distinctive trade dress is important because it helps shield a brand against competition. His arguments focus on the role of packaging in facilitating brand recognition, and 
also on how packaging attributes can prompt symbolic inferences that build positive brand associations (Keller, 1998, pp. 157-172). Although not antithetical to his assertions, our approach is different. We wish to ascertain whether brand cues in the form of differentiated packaging facilitate consumer learning of intrinsic product quality differences among brands. We anticipate that the effects of differentiated packaging should parallel our predictions for differentiated brand names. Specifically:

H2. Differentiated (vs. similar) packaging shapes and colors should yield higher accuracy in memory-based quality judgments.

Associations in memory tend to decay over time, leading to forgetting as well as recall of the wrong targets. These effects can be reduced in two ways. First, strong brand associations should be less vulnerable to decay, because a constant amount of decay should leave a stronger association than it would leave for an association that was weak to begin with (Estes, 1997). In our studies, we contrast two types of learning situations. In the easy learning conditions, consumers taste test products in parallel and can go back and forth between them as they wish. In such conditions, it should be relatively easy to determine differences in taste quality between the products and subjects do not have to rely on brand cues to compare and contrast one product's taste with others experienced previously. As a result, only weak associations should be formed between brand names and taste quality. In difficult learning conditions, consumers taste products sequentially and with significant delays between stimuli. Here, distinguishing among quality differences involves mental comparisons, with brand cues acting as a basis for the recall of prior sensory experiences. Accurate recall may be possible if brand cues are distinctive but not if they are similar. Thus, under difficult learning conditions, memory decay and the decline in recall of experiential targets should be slower for products with differentiated brand cues.

The second way in which the detrimental effects of time on memory for consumption experiences can be mitigated is by creating more differentiated brand associations. For example, Tide and Surf laundry detergents share many intermediary semantic associations. Hence, the differentiation between the two brands depends on a small number of associations, e.g., those related to the mere sound or visual appearance of the names, to distinguish their respective consumption experiences. Decay or perturbation of any of these few non-shared associations may dramatically increase confusion between the consumption experiences of the two brands. In contrast, Tide and Radon differ in almost all their associations. In the case of distinct brand names, decay or perturbation of one non-shared association leaves plenty of others to make sure the appropriate experience is activated by a particular brand name. Thus, again, decay should be reduced and accuracy of recall of experiential targets should stay more stable for products with differentiated brand cues relative to similar brand cues. (Note that this difference may not be apparent when initial recall performance is already poor.) In sum, we hypothesize the following:

H3. Differentiated (vs. similar) brand names should produce a slower decline in accuracy in memorybased quality judgments. ${ }^{1}$

\section{Study 1}

\subsection{Overview}

Study 1 investigated the impact of brand name distinctiveness on accuracy in quality judgments based on consumption experience. Subjects were assigned to one of four conditions and were asked to taste test five stimulus drinks that differed in their objective taste quality. In three conditions, we designed significant delays and interference between the tasting of successive stimuli, making it relatively difficult to learn and remember product quality differences. In two of the three difficult learning conditions, the brand names for the stimuli were designed to be rich in associations and either highly similar or highly differentiated (see below for details). In the third difficult learning condition, the stimuli were simply named by the alphabets "A," "B," "C," etc. so as to have minimal associations. A fourth, easy

\footnotetext{
${ }^{1}$ On account of practical constraints in the presentation of visual cues for delayed recall measures, we could not test similar hypotheses for packaging shape and color.
} 
learning condition was included as a control. Here, we avoided delays and interference by permitting subjects to go back and forth freely between the stimuli before they made quality judgments. Thus, the control condition provided an easy learning environment with no memory demands and the data from this condition enabled us to verify our assumptions regarding objective taste quality. The brand names in this condition were also alphabets. ${ }^{2}$ After a short delay (Time-1) and again after a much longer delay (Time2), we presented the brand names as cues and obtained subjects' judgments of product taste quality.

\subsection{Predictions}

We predicted that accuracy in judgments of taste quality should be relatively high in the easy learning (control) condition at Time-1, because the task did not constrain learning in any way. But in the three difficult learning conditions, delays and interference during learning should impose substantial memory demands even for Time-1 judgments. Based on our preceding conceptualization, the nature of the brand names should be critical in these three conditions.

Accuracy at Time-1 should be relatively low in the difficult learning, alphabet names condition (see previous theorizing). Comparatively, given that the brand names in the similar names condition have strong semantic associations, one might expect this condition to lead to higher accuracy at Time-1. Notwithstanding, these names also should have many overlapping associations, thus leading to a tightly interconnected associative network in memory. These associations, in turn, should be conducive to associative interference in memory and to confusion in judgments (see previous discussion). Hence, on account of countervailing tendencies, we did not expect subjects' accuracy in the similar names condition to be significantly different from the alphabet names condition when learning is difficult. In contrast, subjects in the differentiated names condition should be more accurate in their Time-1 judgments compared to both the alphabet names

\footnotetext{
${ }^{2}$ Because of the considerable time and expense involved in conducting taste tests, we opted for parsimonious designs that enabled us to test our predictions by appropriate planned comparisons of means.
}

(difficult learning) condition and the similar names condition, because the differentiated brand names should have many associations and cause only limited associative interference (H1).

Over time, judgmental accuracy in all four conditions should decline due to retroactive interference. However, in the difficult learning conditions involving (1) alphabet names and (2) similar names, levels of accuracy even at Time-1 should be relatively low. There should be little scope for further decline in accuracy. In contrast, the decline in accuracy between Time- 1 and Time- 2 should be most apparent in the easy learning, alphabet names condition. Although judgmental accuracy initially should be very high in this condition, the lack of strong brand associations should severely impede accurate retrieval of judgments at Time-2. Accuracy in the differentiated names condition should also decline, but at a relatively slower rate, because the stored evaluations have stronger memory associations and less scope for associative interference (H3). Consequently, we predicted a condition $\times$ time crossover interaction such that judgmental accuracy should be higher in the easy learning, alphabet names condition than in the difficult learning, differentiated names condition at Time-1, but the opposite should be true at Time- 2 .

\subsection{Method}

\subsubsection{Subjects}

Subjects were 145 undergraduate marketing students at the University of Florida. They participated for extra credit and were informed that they would be participating in a taste test of tropical fruit juices. Subjects were randomly assigned to one of four experimental conditions. Each session was conducted with up to 8 subjects.

\subsubsection{Stimuli}

The five "brands" of tropical fruit juice (the stimuli in the experiment) were manipulated to differ objectively from one another in taste quality by using the same procedures as Pechmann and Ratneshwar (1992); see also Braun (1999). All stimuli were made from a high quality fresh tropical fruit juice that was available in the local market. We manipulated quality by mixing varying amounts of juice, water, and 
additives across the five stimuli. We selected the following five quality levels: (1) $0 \%$ water, (2) $25 \%$ water, (3) $33 \%$ water, (4) $50 \%$ water, and (5) $50 \%$ water plus one teaspoon of vinegar and one teaspoon of salt per liter.

\subsubsection{Brand name manipulation}

A series of pretests was conducted to select the fictitious brand names for the similar and differentiated names conditions. Our goal was to create brand names that were plausible and fairly appropriate for the category. After considerable pre-testing, we selected the names of five Caribbean islands, namely, Antigua, Aruba, Bahama, Bermuda, and Dominica for the similar names condition and the names Aztec Treasure, Citro, Fresh-Up, Samba, and Tahiti for the differentiated names condition. We conducted a final pretest with 17 subjects who rated (scale of -4 to +4 ) each name with regard to (1) appropriateness for the category of tropical fruit juices, (2) vividness, and (3) implied product quality. Analyses of this pretest data confirmed that the similar (vs. differentiated) names, on average, did not differ significantly with respect to appropriateness for the product category ( $M=.07$ vs. $-.19 ; F(1,16)=1.33, p>.25)$, vividness $(M=.32$ vs. $-.18 ; F(1,16)=1.41 ; p>25)$, and implied quality $(M=-.19$ vs. $-.07 ; F<1)$.

\subsubsection{Procedure}

Upon entering the lab, subjects were led to one of eight cubicles. The cubicles were positioned such that all subjects could see the experimenter, but not each other. On the desk in each cubicle, the subject found a folder with instructions, a large cup of water, and a plate with crackers.

Subjects were asked to carefully read the instructions on the first page of their folders. They were informed that they would be participating in a taste test and that their task was to accurately assess the taste quality of different brands of tropical fruit juices. We also informed subjects that in order to prevent any biases in their judgments, the real brand names of the products would not be revealed. Instead, we would be providing our own labels for the different products. Further, in order to increase motivation for accuracy, subjects were told that the researchers had obtained the ratings of fruit juice experts, and that subjects whose taste quality judgments agreed with those of the experts would enter a lottery with a prize of $\$ 50 .^{3}$ Subjects were then asked to drink the cup of water and to eat a cracker to clear their taste palates.

Subjects in the easy learning (control) condition were then provided cups containing the five juice stimuli. The cups were labeled A through E. Across subjects we used five different assignments of alphabets to the five stimuli for counterbalancing. Subjects tasted all the samples and were allowed go back and forth as they wished between the stimuli. Thus, they were able to compare and contrast their taste impressions in order to arrive at what they considered to be their best possible quality judgments. After spending $5 \mathrm{~min}$ on the taste test, subjects were asked to move on without any delay to the page in the folder that had the dependent measures.

In the three difficult learning conditions, the five juice stimuli were sequentially administered in one randomly selected, but predetermined order of quality to all subjects (see Pechmann \& Ratneshwar, 1992). ${ }^{4}$ Again, across the subjects in each condition, we used five counterbalanced assignments of brand names to the stimuli. The taste test procedure in these conditions was manipulated to impose delays and interference between successive product experiences. Specifically, after they had cleared their taste palates, subjects were first asked to turn to a page in the folder that had the brand name (or alphabet) of the first stimulus in the sequence. At this point, they were also provided with a cup containing the first stimulus. They were asked to drink the whole cup in one or two sips, and to eat a cracker afterwards. After $1 \mathrm{~min}$, they were asked to turn to the next page in the folder, which was blank, and then watch a taped MTV program for five minutes. This procedure of tasting plus waiting was repeated until the subjects had tasted all five stimuli. Five minutes after they had tasted the fifth and final sample, they were asked to turn to the page in the folder containing the dependent measures.

\subsubsection{Dependent measures}

Subjects were first presented a page that listed, in alphabetical order, the same five brand names (or

\footnotetext{
${ }^{3}$ In each of our experiments, the prize was awarded by a random draw among all subjects.

${ }^{4}$ The fixed sequence of objective quality rankings was fourth, first, third, fifth, and second.
} 
alphabets) that they had seen during the tasting phase. They were asked to rank the stimuli from best to worst in taste quality. The Spearman rank correlation (rho) between the subjective taste quality rankings and the objective quality rankings of the five stimuli was later calculated for each subject. This coefficient was used as an index of accuracy in taste quality judgments for Time-1 (see Pechmann \& Ratneshwar, 1992).

Next, subjects were asked to judge taste quality of the five brands on a potentially more sensitive continuous measure. The quality of the best brand (without the name) was shown in the questionnaire by a pre-printed line of $80 \mathrm{~mm}$. With this line length as a benchmark, subjects rated the quality of the brands they judged to be second to fifth in quality by drawing lines of appropriate length. The line lengths were measured later for every subject and used as perceived quality ratings. A quality discrimination index was then constructed for each subject as follows. We computed with appropriate coefficients $(+2,+1,0$, $-1,-2)$ the linear trend in subjects' ratings (i.e., judged line lengths) for the five stimuli when ordered from best to worst on the basis of objective taste quality (see Lynch, Chakravarti, \& Mitra, 1991). This measure was then rescaled as an index ranging from -1 to +1 . The higher the value of the index, the better the subject was able to discriminate in terms of objective taste quality, with maximal quality discrimination among the higher and lower quality brands receiving a score of +1 .

Subsequently, task motivation (Cronbach's $\alpha=.74$ ) and familiarity with the tropical fruit juice category $(\alpha=.95)$ were assessed, each with three-item scales. Then, subjects were asked to guess the purpose of the experiment. None of the answers revealed any insight in the nature of the hypotheses. Finally, we administered the short version of the Cacioppo, Petty, and Kao (1984) instrument for assessing the subject's "need for cognition" $(\alpha=.84)$.

Two weeks after the laboratory session, subjects were contacted by phone for their Time- 2 taste quality judgments. Note that the subjects were not forewarned at Time-1 that they would be contacted again for a second round of judgments. The telephone interviewer reminded subjects about the experiment, repeated the five brand names (or alphabets) in alphabetical order, and asked subjects to rank them again from best to worst in taste quality. The judgmental accuracy index for Time-2 (rho) was computed in the same manner as for Time1. We were able to obtain Time-2 data from 130 of the 145 subjects. Despite repeated efforts, we were unable to reach the others; attrition was fairly even across the four conditions ( 3 to 5 subjects in each case).

\subsection{Results}

The accuracy index (rho) was subjected to a Fisher $r$-to- $z$ transformation for normalization before statistical analysis. We first examined the magnitude of the accuracy index mean in the control condition at Time1 ; its relatively high value $(r h o=84)$ supports our assumption of an objective taste quality ordering among the five stimuli.

The accuracy data for Time- 1 and Time- 2 and the discrimination data for Time-1 were each analyzed by four-condition, one-way ANCOVAs, followed by a priori contrasts (one-tailed tests). A mixed-model MANCOVA was then conducted on the combined accuracy data from Time-1 and Time-2 for the subjects who participated in both phases. To control for possible individual differences, need for cognition, familiarity with tropical fruit juices, and task motivation were used as covariates in all of these analyses. None of these covariates proved to be significant in any of the analyses and hence they are ignored in further discussion. ${ }^{5}$ One subject failed to complete the questionnaire, and was excluded from the analyses. The cell means for the dependent variables are shown in Table 1.

First, we tested for the effectiveness of our manipulation of learning difficulty. As anticipated, at Time-1 subjects in the easy learning, alphabet names (control) condition were more accurate than those in the difficult learning, alphabet names condition $(F(1$, $137)=22.04, p<.001)$. Within the difficult learning conditions, as predicted (H1), at Time-1 subjects in the differentiated names condition were more accurate in their quality judgments than their counterparts in the alphabet names condition $(F(1,137)=4.61$, $p<.05)$. Subjects in the differentiated names condition

\footnotetext{
${ }^{5}$ Analysis of the data without including the covariates yielded the same result.
} 
Table 1

Study 1: accuracy and discrimination in quality judgments as a function of learning condition, brand name differentiation, and time of judgment

\begin{tabular}{lllll}
\hline & \multicolumn{2}{l}{$\begin{array}{l}\text { Easy learning condition with } \\
\text { alphabet names (control) }\end{array}$} & \multicolumn{2}{l}{ Difficult learning conditions } \\
\cline { 3 - 5 } & & Alphabet names & Similar brand names & Differentiated brand names \\
\hline Time-1 Accuracy & $0.84(1.90)$ & $0.38(0.65)$ & $0.47(0.78)$ & $0.64(1.17)$ \\
Time-2 Accuracy & $0.13(0.21)$ & $0.08(0.15)$ & $0.16(0.19)$ & $0.27(0.39)$ \\
Time-1 Discrimination & 0.61 & 0.38 & 0.34 & 0.50 \\
\hline
\end{tabular}

1. Accuracy in quality judgments was assessed for each subject by computing the Spearman rank order correlation (rho) between objective quality and subjective quality rankings. This index measure was subjected to the Fisher $r$-to-z transformation prior to statistical analyses. Table entries show (a) the cell means for the original Spearman coefficients and (b) the cell means for the transformed measure in parentheses.

2. The quality discrimination index was computed via a linear contrast of each subject's quality ratings (theoretical range: -240 to +240 ); this measure was then rescaled to an index ranging from -1 and 1 . See text for details.

3. Time- 1 judgments were obtained in the laboratory at the end of the taste test session. Time- 2 judgments were obtained in telephone interviews 2 weeks after the taste test session. The data for the discrimination measure could not be obtained over the telephone.

4. 46 subjects participated in the easy learning, alphabet names condition; 32 in the difficult learning, alphabet names condition; 31 in the similar brand names condition; and 36 in the differentiated brand names condition.

also performed better than subjects in the similar names condition $(F(1,137)=3.92, p<.05)$. When learning was difficult, the difference in accuracy between subjects in the similar names condition and those in the alphabet names condition was not significant $(F<1)$.

Next, we examined the pattern in judgmental accuracy over time. As anticipated (H3), we found a significant treatment contrast (i.e., easy learning, alphabet names vs. difficult learning, differentiated names) by time (Time-1 vs. Time-2) interaction $(F(1$, $123)=8.74 ; p<.01)$. Paired comparisons showed that at Time-1, on account of the difficult learning experience, subjects in the differentiated names condition performed worse than those in the easy learning, alphabet names condition ( $r h o=.64$ vs. $.84 ; F(1$, 137) $=7.09, p<.01$ ). Notwithstanding, the performance of the former subjects held up better over time (H3): At Time-2, subjects in the difficult learning, differentiated names condition were more accurate in their quality judgments than their counterparts in the easy learning, alphabet names condition $(r h o=.27$ vs. .13; $F(1,123)=8.74, p<0.01)$.

Planned contrasts for the quality discrimination index at Time-1 confirmed those observed for the accuracy measure, with one minor exception. Although directionally consistent, the difference in the discrimination index between the difficult learning, differentiated names condition and the difficult learning, alphabet names condition was not statistically significant $(F(1,137)=2.19, p=.15)$.

\subsection{Discussion}

When differences in true quality were hard to learn, differentiated brand names resulted in better learning and retrieval of the quality experiences associated with various brands. Subjects in the differentiated brand names condition outperformed subjects in both the similar brand names and alphabet names conditions. After a delay, subjects in the differentiated brand names condition outperformed even those in the easy learning, alphabet names condition. Note that a mere retrieval advantage at the point of judgment of the brand names in the differentiated names condition, relative to the similar names and alphabet names conditions, cannot account for our results. We observed improved accuracy and discrimination of taste quality with differentiated names, regardless of the fact that all the brand names were available to subjects both at Time- 1 and Time- 2 . Thus, confusion in brand identity was not an issue because brand names were not the retrieval targets but the directly available cues. The obtained effects also cannot be attributed to particular brand names acting as signals for quality or inferential cues. Although some subjects may have made idiosyncratic quality inferences at the point of judgment based on specific brand names (e.g., "Tahiti is the kind of brand name that suggests good quality"), the counterbalancing of brands and objective quality levels reduces any such effects to mere noise and thus rules out any possibility that our results are due to brand-specific inferences. Our Study 
1 results therefore unambiguously demonstrate the advantage of differentiated brand names in memory for product consumption experiences.

\section{Study 2}

\subsection{Overview}

In Study 2 we tested whether our findings regarding differentiation in brand names could be extended to non-verbal brand elements, particularly the color and shape of a brand's packaging (H2). Subjects were randomly assigned to one of three experiment conditions: no visual differentiation (control), differentiated packaging shapes, and differentiated packaging colors. All three conditions involved the difficult learning task and the set of similar brand names (Caribbean islands) used in Study 1. In the differentiated colors condition, the five juice samples were each presented with a differently colored picture of a juice bottle. In the differentiated shapes condition, color was held constant, but bottle shape was varied across stimuli. Finally, in the control condition, both bottle color and shape were held constant across stimuli.

\subsection{Predictions}

In all three conditions delays and interference during learning should impose substantial memory demands. We predicted that increased differentiation in packaging colors or shapes should improve accuracy in memory-based quality judgments, relative to the control condition in which visual differentiation was minimal.

\subsection{Method}

Subjects were 192 undergraduates from the same subject pool as Study 1 and they were randomly assigned to the three experiment conditions. As in Study 1, they participated in a taste test of five tropical fruit juices. They were told that the juices were either on the local market or to be introduced soon, but that labels to disguise the brand's identities had replaced the original brand names. In addition, they would see, "as is common in standard commercial taste test procedures," a similarly disguised picture of the packaging. In the control condition, the bottle was identical for all brands: a standard shape was used and all bottles were colored yellow. In the color differentiation condition, the bottle shape was held constant, but all the bottles had different colors. The three primary (yellow, red, and blue) and two secondary colors (purple, green) from the color wheel were chosen for maximal color differentiation. In the shape differentiation condition, color was held constant but all the bottles had different shapes. We picked five bottle shapes that were in commercial use for juice drinks or related products. The bottle shapes are depicted in Fig. 1. The brand names, in a neutral type font, were superimposed on the bottles. The assignment of packaging characteristics to brand names was held constant, but the assignment of name-package combinations to objective quality levels was counterbalanced across subjects. In this study, only Time-1
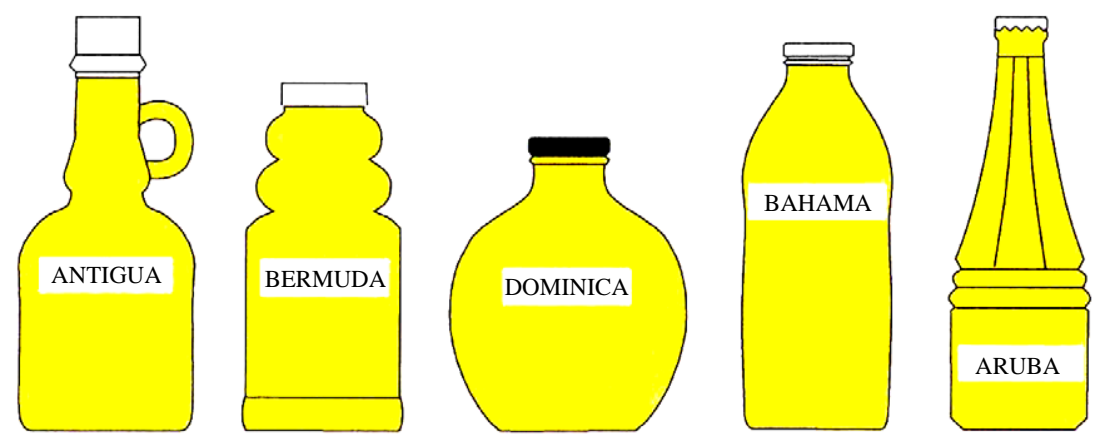

Fig. 1. Study 2 stimuli: differentiated packaging shapes. 
quality rankings and ratings were obtained. Subjects made these taste quality judgments on a page that depicted the five bottle-brand name combinations that they had seen in the tasting phase. All other aspects of Study 2 were identical to Study 1.

\subsection{Results}

As in Study 1, none of the subjects indicated any insight in the nature of the experimental manipulations. The accuracy and discrimination indices were subjected to three condition one-way ANCOVAs, followed by a priori contrasts. Need for Cognition $(\alpha=.86)$, product familiarity $(\alpha=.95)$, and task motivation $(\alpha=72)$ were introduced as covariates. None of these covariates proved significant in the analyses, and hence they are ignored in further discussion. Means for all conditions are shown in Table 2.

Planned contrasts indicated that relative to the control group in which there was no visual differentiation, the differentiated packaging shapes condition resulted in a marginal improvement in accuracy in quality judgments $(F(1,186)=2.86, p<.10)$. In the differentiated colors condition, accuracy was significantly better than in the control group $(F(1$, $186)=5.47, p<.05)$. The results for the quality discrimination index followed the same general pattern; the planned contrasts for differentiated packaging shapes as well as differentiated packaging colors yielded reliable differences against the control condition $(p$ 's<.05).

Table 2

Study 2: accuracy and discrimination in quality judgments as a function of packaging differentiation

\begin{tabular}{llll}
\hline & \multicolumn{2}{l}{ Packaging condition } & \\
\cline { 2 - 4 } & $\begin{array}{l}\text { No visual } \\
\text { differentiation } \\
\text { (control) }\end{array}$ & $\begin{array}{l}\text { Differentiated } \\
\text { shapes }\end{array}$ & $\begin{array}{l}\text { Differentiated } \\
\text { colors }\end{array}$ \\
\hline Accuracy & $0.44(0.72)$ & $0.58(0.96)$ & $0.58(1.05)$ \\
Discrimination & 0.31 & 0.43 & 0.41 \\
\hline
\end{tabular}

1. The index measures for accuracy and discrimination in quality judgments were constructed as in Study 1; see Table 1 notes.

2. All three conditions involved difficult learning and similar brand names. Only Time-1 judgments were obtained in this study.

3. 64 subjects participated in the no visual differentiation condition; 62 in the differentiated shapes condition; and 66 in the differentiated colors condition.

\subsection{Discussion}

The results confirm that even when brand names are highly similar, differentiation in packaging shapes and colors can enhance accuracy in memory-based quality judgments. Thus, as in the case of distinctive brand names, visual differentiating cues facilitate the encoding and retrieval of quality differences that are based on consumption experiences. These results cannot be attributed to inherently better memory for the visually differentiated packages themselves, because subjects were provided with all of the packaging stimuli cues when they made their quality judgments. Also, because visual packaging cues and objective quality were completely counterbalanced, the results cannot be accounted for by potential biases in quality perception induced by specific packaging shapes or colors.

\section{Study 3}

In Studies 1 and 2, we did not include in the learning environment an important extrinsic cue, namely price. Price and objective quality in the market are often poorly correlated (Oxenfelt, 1950). Nevertheless, it has been shown that consumers often rely on price to infer product quality, especially in learning environments that provide little opportunity for diagnostic product experience (Broniarczyk \& Alba, 1994; Pechmann \& Ratneshwar, 1992; Rao \& Monroe, 1988). Such effects may be even stronger when quality information is experiential and, hence, inherently subjective and malleable. And when consumers rely on price cues in making quality judgments in situations where there is little or no correlation between price and true quality, they are obviously likely to be quite inaccurate in their judgments.

Hence the question: Can differentiation in extrinsic cues such as brand names and packaging facilitate accuracy in memory-based quality judgments even when product prices provide potentially misleading cues to quality? Note that when consumers are confronted with a difficult learning task, there may be a strong tendency to rely only on simple pricequality inferences. It is hence unclear whether the beneficial effects we found for distinctive brand cues 
in Studies 1 and 2 would survive in the face of price cues. Accordingly, in Study 3 we explored the role of distinctive brand cues in a manner similar to Studies 1 and 2 , except that we included product price as an additional, but potentially deceptive, extrinsic cue in the environment.

A secondary objective in Study 3 was to verify whether the effects of distinctive brand cues would be found when cue distinctiveness involves a combination of cues rather than a single cue. Note that in Study 1 we investigated differentiation in brand names, but not in product packaging. In Study 2, we held the set of brand names constant across conditions, and we varied either packaging shapes or packaging colors. In the marketplace, however, a distinctive trade dress typically involves a unique combination of all of the aforementioned attributes (Keller, 1998). Would the results observed in the first two studies extrapolate to situations where products in a category are differentiated simultaneously in several extrinsic cues? This question is important because there is some evidence that consumers find it difficult to learn quality differences in multiple-cue situations on account of information complexity and overload (Hutchinson \& Alba, 1991). In Study 3, we hence decided to test the generalizability of the previous findings by concurrently differentiating brand names, packaging shapes, and packaging colors.

\subsection{Overview}

Study 3 had four experiment conditions, one with an easy learning task and three with a difficult learning task. Subjects in the easy learning (control) condition were exposed to a set of similar brand names and packaging and also saw price information for each brand. As in Study 1, these subjects received all the juice stimuli at the same time and could go back and forth to compare and contrast their taste experiences. The three difficult learning conditions were as follows: (1) similar brand names and packaging, no price information, (2) similar brand names and packaging, with price information, and (3) differentiated brand names and packaging, with price information. Subjects in all three difficult learning conditions experienced sequential stimuli presentation and also delays and interference between successive stimuli, as in Study 1. In the three experiment conditions where subjects received price information, prices were assigned to the stimuli such that the rankorder correlation between price and objective taste quality was zero.

The taste test tasks, instructions to subjects, and dependent measures were very similar to the procedures followed in Studies 1 and 2. We however made a change in the product category to suit the local conditions of Study 3 (see below). Further, as in Study 1 but unlike Study 2, we were able to obtain both immediate and delayed judgments of product quality from our subjects. Subjects judged the stimuli twice, first after the learning phase ("Time-1"), and again during a second lab session two weeks later ('Time-2"). By bringing subjects back to the lab for Time-2, we were able to gather delayed measures for both the accuracy and the discrimination indices (cf. Study 1). Subjects did not know that we would be asking them to judge the stimuli again at Time-2 and thought that they would be coming back for an unrelated experiment.

\subsection{Predictions}

We predicted that accuracy and discrimination should be high in the easy learning (control) condition, regardless of similar brand names and packaging as well as potentially misleading price information, because (1) the learning environment imposes no memory demands, and (2) given diagnostic product experience, subjects are unlikely to rely on price-quality inferences. The effect of price-quality inferences however should be evidenced in a comparison of Time-1 judgments in the two conditions that involved difficult learning and similar brand names and packaging. Note that these conditions were identical in design variables, other than the presence versus absence of price information. Given that a difficult learning task may cause subjects to rely on a price-quality cue, and given that the price information in the experiment is actually uncorrelated with objective quality, accuracy at Time-1 should be significantly lower in the similar brand names and packaging condition where price information is provided.

The effects of distinctive brand cues on accuracy in Time-1 judgments should be apparent in a comparison 
of the two difficult learning conditions where subjects are provided with price information. Compared to their counterparts in the similar brand names and packaging condition, subjects in the differentiated brand names and packaging condition should be able to encode their product experiences with richer and less overlapping sets of brand associations, thereby reducing memory interference and reducing the need to rely on a price-quality cue. Consequently, the latter subjects should be more accurate in their initial judgments.

As in Study 1, we expected judgmental accuracy and discrimination to decline over time. This decline should be most apparent in the easy learning, similar brand names and packaging condition, where initial accuracy is expected to be quite high. In contrast, quality judgments should be most stable in the differentiated brand names and packaging condition. Accordingly, we predicted a treatment condition by time crossover interaction such that accuracy at Time1 should be higher in the easy learning, similar brand names and packaging condition than in the difficult learning, differentiated brand names and packaging condition, while this pattern should be reversed at Time-2. That is, accuracy in quality judgments may decline over time in both of these conditions, but far less rapidly when subjects have encoded their experiences on the basis of differentiated brand names and packaging.

\subsection{Method}

\subsubsection{Subjects}

Subjects were 154 undergraduate students at the K.U. Leuven. They participated as part of the requirements for an undergraduate marketing class, and were told that they would take part in a taste test of fruit juices. Sessions were conducted with groups of up to nine participants. The experiment was conducted in two sessions, with the second one exactly two weeks after the first. Nineteen subjects failed to return for the second session. Subject attrition was uniform across conditions.

\subsubsection{Stimuli}

Because the tropical fruit juice product utilized in Study 1 was not available in Belgium, we used orange juices as the stimuli. Using a high-quality, fresh orange juice as the base, we created five objective quality levels by diluting it with different amounts of water: $0 \%$ water, $25 \%$ water, $33 \%$ water, $50 \%$ water and $67 \%$ water.

\subsubsection{Brand name and packaging manipulations and pretests}

Two new sets of brand names were selected after pretests that established their meaningfulness to local subjects. The similar brand names were names of five Spanish cities or regions, namely Cordoba, Cataluna, Cartagena, Almazora, and Almarcha. The differentiated brand names were Cordoba, Olé-Olé, Vers Geperst, Appeltje-van-Oranje, and Aztec Treasure. A final pretest with 35 subjects confirmed that the similar (vs. differentiated) names, on average, did not differ significantly with respect to category appropriateness $(M=-.43$ vs. $-.02 ; F(1,34)=1.39$, $p>.25)$, vividness $(M=.18$ vs. $.56 ; F(1,34)=1.13$; $p>.25$, or implied quality $(M=.08$ vs. $-.01 ; F<1)$. In the differentiated brand names and packaging condition, we combined the aforementioned differentiated names with both the differentiated bottle shapes and the differentiated packaging colors used in Study 2. In the similar brand names and packaging conditions, packaging shape and color were held constant.

\subsubsection{Procedure}

Subjects were randomly assigned to one of four experimental conditions. The study was presented as a taste test of new juice drinks. They were promised participation in a lottery of $€ 40$ if their evaluations conformed to those of a panel of expert judges. The procedure and dependent measures were identical to Studies 1 and 2, except as noted here. In the three conditions where price information was provided to subjects, a price label was put on each brand. We designed the price range across the five stimuli to reflect local market conditions. Prices were assigned to the samples such that the rank-order correlation between prices and taste quality was zero. Assignment of brand name/price combinations to stimuli was counterbalanced across subjects. As in Study 1, task motivation $(\alpha=.95)$ and familiarity with the product category $(\alpha=.71)$ were measured at Time-1; but in this study we were unable to measure "Need for Cognition." The second session (Time-2) was conducted in the lab and exactly two weeks later. In this 
session, subjects were presented with the brand name and packaging representations of the original stimuli and asked again for their quality ranking and ratings. After they completed these Time- 2 measures, they moved on to a previously announced (unrelated) experiment.

\subsection{Results}

The accuracy (after $r$-to-z transformation) and discrimination data for Time-1 were analyzed by four-group one-way ANCOVAs, followed by a priori contrasts. A mixed model MANCOVA was conducted on the combined Time- 1 and Time- 2 data for the 135 subjects who participated in both sessions. Familiarity with orange juice and task motivation were included as covariates in these analyses, but proved not significant. Cell means are presented in Table 3.

At Time-1, as anticipated, subjects in the easy learning, similar brand names and packaging, with price information condition proved to be quite accurate in their quality judgments (mean $r h o=.94$ ); this data point supports the presumed objective quality ordering among the stimuli. Next, we assessed the impact of price cues on accuracy through a planned comparison of the difficult learning, similar brand names and packaging, with price information condition and the difficult learning, similar brand names and packaging, no price information condition. This contrast proved to be significant $(F(1,148)=3.00$, $p<.05)$ such that subjects were less accurate in their Time-1 quality judgments when they were exposed to price (vs. no price) information ( $r h o=.25$ vs. .50 ).
The critical test for the effects of distinctive brand cues on accuracy in this study is the contrast at Time-1 between the difficult learning, differentiated brand names and packaging, with price information condition and the difficult learning, similar brand names and packaging, with price information condition (columns 4 and 3, data row 1, of Table 3). Indeed, accuracy was significantly higher among subjects who were exposed to differentiated (vs. similar) brand names and packaging $(r h o=.50$ vs. $.25 ; F(1,148)=$ $7.38, p<.01$ ). Finally, we expected accuracy in quality judgments to hold up better over time when there is differentiation in brand names and packaging. The MANCOVA results confirmed a treatment contrast (easy learning, similar brand names and packaging, with price information condition vs. difficult learning, differentiated brand names and packaging, with price information condition) by time (Time-1 vs. Time-2) crossover interaction $(F(1,129)=50.4, p<.001)$. Follow-up comparisons confirmed that the trends in accuracy over time were as predicted. At Time-1, subjects in the difficult learning, differentiated brand names and packaging, with price information condition were considerably less accurate than their counterparts in the easy learning, similar brand names and packaging, with price information condition $(r h o=.50$ vs. $.94 ; F(1,134)=41.3, p<.001)$. At Time2 , however, this pattern was reversed; subjects in the difficult learning, differentiated brand names and packaging condition were more accurate than those who had an easy learning task, but encountered similar brand names and packaging ( $r h o=.49$ vs. $.29 ; F(1,129)=3.41, p<.10)$.

Table 3

Study 3: accuracy and discrimination as a function of learning condition, brand name and packaging differentiation, price information, and time of judgment

\begin{tabular}{|c|c|c|c|c|}
\hline & \multirow{2}{*}{$\begin{array}{l}\text { Easy learning condition } \\
\text { Similar brand names } \\
\text { and packaging }\end{array}$} & \multicolumn{3}{|c|}{ Difficult learning conditions } \\
\hline & & $\begin{array}{l}\text { Similar brand names } \\
\text { and packaging }\end{array}$ & $\begin{array}{l}\text { Similar brand names } \\
\text { and packaging }\end{array}$ & $\begin{array}{l}\text { Differentiated brand } \\
\text { names and packaging }\end{array}$ \\
\hline Price information & Yes & No & Yes & Yes \\
\hline Time-1 Accuracy & $0.94(2.40)$ & $0.50(0.75)$ & $0.25(0.41)$ & $0.50(0.94)$ \\
\hline Time-2 Accuracy & $0.29(0.51)$ & $0.18(0.32)$ & $0.24(0.31)$ & $0.49(0.94)$ \\
\hline Time-1 Discrimination & 0.66 & 0.33 & 0.18 & 0.38 \\
\hline Time-2 Discrimination & 0.20 & 0.15 & 0.15 & 0.28 \\
\hline
\end{tabular}

1. The index measures for accuracy and discrimination in quality judgments were constructed as in Study 1; see Table 1 notes.

2. Accuracy and discrimination at Time-2 were assessed during a second lab session, two weeks after Time-1.

3. 46 subjects participated in the easy learning condition, and 36 in each of the three difficult learning conditions. 
In separate analyses we verified that all of the above results on the accuracy index were replicated on the quality discrimination index. The only exception was that the Time- 2 contrast between the difficult learning, differentiated brand names and packaging condition and the easy learning, similar brand names and packaging condition was in the right direction but not statistically significant ( $M=.28$ vs. .20, $n s)$.

\subsection{Discussion}

These results, obtained with a different product and in a different part of the world, supported and extended those of the previous two experiments. In particular, we found that distinctive brand cues improve accuracy and discrimination in quality judgments even when (1) price cues are deceptive in regard to quality inferences and (2) there is simultaneous differentiation in multiple cues such as brand names, packaging shapes, and packaging colors. Inspection of the means in the three difficult-learning conditions of Table 3 suggests initial (Time-1) accuracy and discrimination in the similar brand names and packaging conditions were reduced significantly on account of deceptive price cues. But differentiated brand names and packaging helped restore accuracy and discrimination to approximately the levels that prevailed when price information was not provided. Further, two weeks after the learning phase, subjects in the differentiated brand names and packaging condition exhibited a level of accuracy in quality judgments that was about $100 \%$ better than the average accuracy in the other three conditions.

\section{General discussion}

Prior to our work, several researchers have shown how extrinsic cues such as brand names (e.g., Allison \& Uhl, 1964; Lee \& Ang, 2003), advertising claims (e.g., Braun, 1999; Hoch \& Ha, 1986), prices (e.g., Rao \& Monroe, 1988), and "irrelevant" product attributes (e.g., Carpenter, Glazer, \& Nakamoto, 1994) can lead to biased product evaluations and sub-optimal choices by consumers. These findings may easily be taken to suggest that differentiation tactics that are unrelated to product performance stand in the way of consumers who wish to learn about true quality differences among competing products through their consumption experience. However, most of these prior studies used scenarios in which (1) there were minimal intrinsic differences among products, (2) consumers were exposed to all products more or less simultaneously, or (3) there were insignificant delays between product exposure and quality judgments. Notwithstanding, many realworld consumption situations are characterized by at least some intrinsic differences in quality, by sequential instead of simultaneous product exposure, and by significant delays between product exposure and quality judgments. Our three studies suggest that in such everyday situations, extrinsic cues such as distinctive brand names and packaging will often increase the accuracy of consumers' memory-based product evaluations and, hence, potentially increase consumer welfare.

Extrinsic cues that are present during a consumption episode provide retrieval pathways in associative memory to the original perceptual experience when consumers later need to remember product quality. Every distinctive extrinsic cue that is present both at learning and at recall provides an additional retrieval cue to the consumption experience, increasing the probability that the original experience will be remembered accurately. Thus, differentiated brand names and packaging are conducive to singular associations and unique memory pathways to individual product quality experiences. In contrast, similarity in brand names and packaging cues leads to overlapping associations and memory interference when consumers attempt to learn and remember quality differences. Further, this beneficial effect of distinctive brand cues in facilitating accuracy in memory-based quality judgments becomes increasingly more apparent with the passage of time.

\subsection{Memory-based judgments in consumer decision-making}

Our findings build on prior consumer research that has investigated the processes involved in memorybased judgments in consumer decision-making (e.g., Alba et al., 1991; Baker, 2001; Pham \& Johar, 1997). This literature usually assumes that consumers trying to decide which product to buy from several 
alternatives on a store shelf will only make inferences on the basis of extrinsic cues if they cannot directly retrieve intrinsic quality attributes. But the results of Study 3 showed that in the two conditions that had similar brand names and packaging, subjects who were provided with price information were less accurate in their quality judgments than their counterparts in the no-price condition. These data seem to indicate that subjects who might correctly remember taste experiences if they used a recall-based strategy were, in fact, basing their judgments at least partially on a price-quality inference process. Our results hence suggest some use of inference processes even when direct memory retrieval processes are feasible. This makes sense if one assumes that a cue-based inference process, though more effortful than pure guessing, is less effortful than retrieval from memory in situations where the latter process is fraught with associative interference from competing stimuli.

Taken as a whole, the implication of our findings is that for some consumers (e.g., those who lack requisite product expertise or motivation), or in certain learning situations (e.g., when a person's product or service experiences are very ambiguous), extrinsic cues such as brand names and packaging attributes might primarily serve as a basis for quality inferences, whereas for other consumers or in other learning situations these cues may mainly act as "memory pegs" for effective learning and retrieval of past quality experiences. Future research may be able to further clarify such multiple roles of extrinsic cues in quality judgments and may also illuminate the factors that moderate their prevalence and relative influence.

\subsection{Managing brand equity}

The present findings also shed new light on the role of differentiation as an important element of brand equity. In the brand equity literature, differentiation of brand associations is thought to be important because a brand name that is linked to unique consumer benefits gains a sustainable competitive advantage (Keller, 1998). Such "unique selling propositions" provide consumers with reasons to choose one product over another.

Our research, however, suggests that in addition to benefit differentiation, differentiation in extrinsic brand cues is also quite important. Extrinsic brand cues such as the unique semantic and phonetic aspects of a brand name and the visual elements of brand packaging refer to a brand's trademark and trade dress (Jacoby \& Morrin, 1998; Peterson, Smith, \& Zerillo, 1999). Previous discussions of the role of trade dress in brand equity have focused on factors such as ease of brand recognition and the need for symbolic associations that reinforce a brand's overall positioning (Keller, 1998). But our findings suggest that, in addition, differentiated brand name and packaging fundamentally help consumers to learn and accurately remember a product's intrinsic quality. By facilitating the learning and retrieval of intrinsic quality differences among competing products, extrinsic brand cues can be an important source of brand equity for high-quality brands. Indeed, marketers of superior quality products may wish to encourage consumers to frequently process extrinsic brand cues while they are actually consuming the product. Tactics that are appropriate for this objective include consumer promotions where cups and bowls with brand names and logos are given away for products such as coffee and breakfast cereals, or TV commercials for beer and carbonated beverages that subtly encourage consumers to "drink from the bottle".

\subsection{Trademark dilution: public policy and legal implications}

Carpenter and Nakamoto (1989) suggest that when late entrants in a product category copy the features of pioneers, consumers usually do not evaluate the former favorably. Notwithstanding, copycat brands (e.g., those that mimic the name and packaging of major national brands) continue to proliferate in the consumer market (Jacoby \& Morrin, 1998). Our findings provide at least one rationale for their success.

When extrinsic cues such as packaging colors and designs are very similar, and when products are purchased and consumed sequentially over an extended period of time, it becomes very difficult for consumers to learn and remember quality differences (assuming such differences exist) between wellestablished brands and lower-priced copycats. Further, thanks to similarities in brand name and packaging, 
consumers who are considering whether to buy a lowpriced copycat may mistakenly think that they have had a satisfactory prior consumption experience with that brand, when that particular experience may have actually occurred with the established brand that has been copied. Similarly, the extrinsic aspects of the high-quality brand might lead consumers to retrieve from memory unsatisfactory prior experiences that in reality correspond to a lower-quality, copycat brand. Both of these sources of confusion provide a market advantage to the copycat, one that it would not enjoy if it had a differentiated brand name or visual appearance.

We note that the type of confusion we are referring to here is quite different from the one where consumers simply mistake the identity of the brand or the manufacturer at the point of purchase (Jacoby \& Morrin, 1998; Simonson, 1993). For example, Jacoby and Morrin (1998) discuss U.S. trademark litigation involving Vaseline Intensive Care Lotion and a look-alike product called Venture and also the case of two similar progestin-based drugs for women named Provera and Prempro. In the context of the present research the issue is not whether consumers buy Venture just because they mistake it on the store shelf for Vaseline. Rather, our findings speak to the possibility that similarity in brand names and visual similarity in packaging can be conducive to confusion in memory regarding one's past experiences and perceptions of quality regarding competing products.

Thus, although it is clear to consumers that Cataluna is not the same brand of orange juice as Almarcha, they may still confuse the experiences they have had with each of these distinct products on account of semantic and phonetic similarity. Consumer confusion may originate not at the level of misidentification of the brand per se, but at the level of confusing the experiences or benefits provided by each brand. Such confusion may contribute to exaggerated quality perceptions for the copycat, thereby damaging not only the copied brand, but other brands in the marketplace as well (Warlop \& Alba, 2004). Our results hence suggest that it is important to include such benefit confusion in addition to brand confusion in framing legislation and public policy regarding trade dress imitation.
In this context it is interesting to note that in the U.S. The Federal Trademark Dilution Act (FTDA) of 1995 considerably expanded the scope of legal protection for established (or "famous") brands beyond the Lanham Act of 1946 (see Peterson et al., 1999 for a detailed discussion). The FTDA defines dilution as "the lessening of the capacity of a famous mark to identify and distinguish goods and services, regardless of [...] likelihood of confusion, mistake or deception" (Peterson et al., 1999, p. 258). Thus, in terms of the FTDA's definition, trademark dilution is not limited to sheer confusion of look-and sound-alike brands. Our findings suggest that mental mix-ups of the experiences and benefits provided by famous brands and their not-so-famous copycats may be a type of trademark dilution for which aggrieved parties in the U.S. can seek legal remedies. Whereas the law in some European countries (e.g., the 1971 Benelux Trademarks Act) has provided similar scope of protection, the 1988 European Directive for the Harmonization of Trade Mark Laws is formulated and interpreted more conservatively, and tends to limit protection to instances of actual confusion of identity (for a recent review, see Casparie-Kerdel, 2001).

\subsection{Limitations and future research}

Some limitations need to be addressed. Although our studies provided strong evidence for the effects of brand name differentiation, our manipulation of this variable was not sufficiently fine-grained to separate semantic and non-semantic effects. The semantically similar set of brand names was also acoustically and visually more similar. Future research may be able to better isolate the roles of semantic vs. non-semantic differences among brand names, logos and other visual packaging cues. Past memory research suggests that semantic differentiation may indeed prove to be the most important. For example, using a paired-associate learning paradigm, Underwood, Ham, and Ekstrand (1962) manipulated visual and semantic differentiation of words that served as retrieval cues in a memory test. The semantic variable was much more influential, suggesting that it had been spontaneously selected as the more effective part of the retrieval cue. In terms of consumer learning, this effect might be compounded by the likely consumer belief that brand names 
identify different choice alternatives uniquely, while packaging colors and shapes often do not.

A further limitation is that we studied the effects of distinctive brand cues in combination with price as a potentially biasing cue, but did not empirically examine the joint influence of brand cue differentiation with other marketing variables such as advertising claims or quality inferences engendered by specific brand names. Brand and price cues-and any other cues for which consumers maintain a priori beliefs regarding relationships with product qualitymay operate both as inference-producing cues and as differentiating features. Future research may be able to provide many more interesting insights on this issue and, more generally, on whether, when, and why extrinsic cues facilitate or impede consumer learning of intrinsic quality differences.

\section{Acknowledgements}

The authors gratefully acknowledge the assistance of Agnes Moors in collecting the data. They also thank Mike Barone, Siegfried Dewitte, Davy Lerouge, Kevin Keller, Maureen Morrin, Girish Punj, Wouter Vanhouche, Vinciane Vanovermeire, and seminar participants at Baruch College, Florida International University, Rutgers University (Camden) and University of Connecticut for their helpful comments.

\section{References}

Alba, J. W., Hutchinson, J. W., \& Lynch, J. G., Jr. (1991). Memory and decision making. In T.S. Robertson, \& H.H. Kassarjian (Eds.), Handbook of consumer behavior (pp. 1-19). Englewood Cliffs, NJ: Prentice-Hall.

Allison, R. I., \& Uhl, K. P. (1964). Influence of beer brand identification on taste perception. Journal of Marketing Research, 1(3), 36-39.

Baker, W. E. (2001). The diagnosticity of advertising generated brand attitudes in brand choice contexts. Journal of Consumer Psychology, 11(2), 129-139.

Braun, K. A. (1999). Post-experience advertising effects on consumer memory. Journal of Consumer Research, 25(4), 319-334.

Broniarczyk, S. M., \& Alba, J. W. (1994). Theory versus data in prediction and correlation tasks. Organizational Behavior and Human Decision Processes, 57(1), 117-139.
Burke, R. R., \& Srull, T. K. (1988). Competitive interference and consumer memory for advertising. Journal of Consumer Research, 15(1), 55-68.

Cacioppo, J. T., Petty, R. E., \& Kao, C. F. (1984). The efficient assessment of need for cognition. Journal of Personality Assessment, 48(3), 306-307.

Carpenter, G. S., Glazer, R., \& Nakamoto, K. (1994). Meaningful brands from meaningless differentiation: The dependence on irrelevant attributes. Journal of Marketing Research, 31(3), $339-350$.

Carpenter, G. S., \& Nakamoto, K. (1989). Consumer preference formation and pioneering advantage. Journal of Marketing Research, 26(3), 285-298.

Casparie-Kerdel, S. (2001). Dilution disguised: Has the concept of trade mark dilution made its way into the laws of Europe? European Intellectual Property Review, 23(2), 185-195.

Chattopadhyay, A., \& Alba, J. W. (1988). The situational importance of recall and inference in consumer decision making. Journal of Consumer Research, 15(1), 1-13.

Erdem, T., \& Swait, J. (1998). Brand equity as a signaling phenomenon. Journal of Consumer Psychology, 7(2), 131-157.

Estes, W. K. (1997). Processes of memory loss, recovery, and distortion. Psychological Review, 104(1), 148-169.

Henderson, P. W., Cote, J. A., Leong, S. M., \& Schmitt, B. (2003). Building strong brands in Asia: Selecting the visual components of image to maximize brand strength. International Journal of Research in Marketing, 20(4), 297-314.

Hoch, S. J., \& Deighton, J. (1989). Managing what consumers learn from experience. Journal of Marketing, 53(2), 1-20.

Hoch, S. J., \& Ha, Y. W. (1986). Consumer learning from advertising and the ambiguity of processing experience. Journal of Consumer Research, 13(2), 221-233.

Hutchinson, J. W., \& Alba, J. W. (1991). Ignoring irrelevant information: Situational determinants of consumer learning. Journal of Consumer Research, 18(3), 325-345.

Jacoby, J., \& Morrin, M. (1998). 'Not manufactured or authorized by...': Recent federal cases involving trademark disclaimers. Journal of Public Policy and Marketing, 17(1), 97-107.

Keller, K. L. (1991). Memory and evaluations in competitive advertising environments. Journal of Consumer Research, 17(4), 463-476.

Keller, K. L. (1998). Strategic brand management. Upper Saddle River, NJ: Prentice-Hall.

Kirmani, A., \& Rao, A. R. (2000). No pain, no gain: A critical review of the literature on signaling unobservable product quality. Journal of Marketing, 64(2), 66-79.

Krishnan, H. S. (1996). Characteristics of memory associations: A consumer based brand equity perspective. International Journal of Research in Marketing, 13(4), 389-406.

Kumar, A. (2000). Interference effects of contextual cues in advertisements on memory for ad content. Journal of Consumer Psychology, 9(3), 155-166.

Lee, Y. H., \& Ang, K. S. (2003). Brand name suggestiveness: A Chinese language perspective. International Journal of Research in Marketing, 20(4), 323-336.

Lynch, J. G., Jr., Chakravarti, D., \& Mitra, A. (1991). Contrast effects in consumer judgments: Changes in mental representa- 
tion or in the anchoring of rating scales? Journal of Consumer Research, 18(2), 284-297.

McClelland, J. L. (2000). Connectionist models of memory. In E. Tulving, \& F. I. M. Craik (Eds.), The Oxford handbook of memory (pp. 583-596). Oxford: Oxford University Press.

Oxenfelt, A. R. (1950). Consumer knowledge: Its measurement and extent. Review of Economics and Statistics, 32(4), 300-314.

Paivio, A. (1971). Imagery and verbal processes. New York: Holt, Rinehart \& Winston.

Pechmann, C., \& Ratneshwar, S. (1992). Consumer covariation judgments: Theory or data driven? Journal of Consumer Research, 19(3), 373-386.

Peterson, R. A., Smith, K. H., \& Zerillo, P. C. (1999). Trademark dilution and the practice of marketing. Journal of the Academy of Marketing Science, 27(2), 255-268.

Pham, M. T., \& Johar, G. V. (1997). Contingent processes of source identification. Journal of Consumer Research, 24(3), 249-265.

Rao, A. R., \& Monroe, K. B. (1988). The moderating effect of prior knowledge on cue utilization in product evaluations. Journal of Consumer Research, 15(2), 253-264.

Resnik, A., \& Stern, B. L. (1977). An analysis of the information content in television advertising. Journal of Marketing, 41(1), $50-53$.
Sen, S. (1999). The effects of brand name suggestiveness and decision goal on the development of brand knowledge. Journal of Consumer Psychology, 8(4), 431-455.

Simonson, A. F. (1993). How and when do trademarks dilute?: A behavioral framework to judge "likelihood of dilution". Trademark Reporter, 83(2), 149-174.

Slamecka, N. J., \& Graf, P. (1978). The generation effect: Delineation of a phenomenon. Journal of Experimental Psychology. Learning, Memory, and Cognition, 4(6), 592-604.

Smith, R. E., \& Swinyard, W. R. (1982). Attitude-behavior consistency: The impact of product trial versus advertising. Journal of Marketing Research, 20(3), 257-268.

Underwood, B. J., Ham, M., \& Ekstrand, B. R. (1962). Cueselection in paired associate learning. Journal of Experimental Psychology, 64(4), 405-409.

Warlop, L., \& Alba, J. W. (2004). Sincere flattery: Trade-dress imitation and consumer choice. Journal of Consumer Psychology, 14(1 and 2), 21-26.

Wright, A. A., \& Lynch, J. G., Jr. (1995). Communication effects of advertising versus direct experience when both search and experience attributes are present. Journal of Consumer Research, 21(4), 708-719. 